\title{
Entrelacs
}

Cinéma et audiovisuel

12 | 2016

Nouvelles formes audiovisuelles documentaires

\section{Man on Bridge : une forme qui échappe aux catégories.}

Rémy Besson

\section{OpenEdition}

Journals

Electronic version

URL: http://journals.openedition.org/entrelacs/1878

DOI: $10.4000 /$ entrelacs. 1878

ISSN: 2261-5482

\section{Publisher}

Éditions Téraèdre

Electronic reference

Rémy Besson, « Man on Bridge : une forme qui échappe aux catégories. », Entrelacs [Online], 12 | 2016, Online since 14 January 2016, connection on 19 April 2019. URL : http://journals.openedition.org/ entrelacs/1878; DOI : 10.4000/entrelacs.1878

This text was automatically generated on 19 April 2019

Tous droits réservés 


\title{
Man on Bridge : une forme qui échappe aux catégories.
}

\author{
Rémy Besson
}

\section{Introduction}

1 Jusqu'à la fin du vingtième siècle, il a été relativement aisé de distinguer un fonds d'archives visuelles d'un film documentaire portant sur le passé. Les archives constituaient alors une des sources que le réalisateur et/ou ses assistants consultaient lors de leurs recherches iconographiques (Morrissey, 2004). Elles s'intégraient ensuite à un processus de mise en récit complexe (note d'intention, scénario, tournage, montage). Seule l'équipe du film allait donc en archives, le public ayant lui accès à une forme médiatisée de celles-ci. Parfois, un corpus singulier d'images devenait l'objet d'un film portant sur l'histoire du visuel (Delage et Guigueno, 2004), mais cela restait rare. Sources ou objets, les documents archivés étaient considérés comme des traces du passé servant de base à une représentation de l'histoire, le film (Véray, 2011).

2 L'avènement de l'ère du numérique qui est une « rupture, non seulement technologique, mais encore anthropologique et culturelle de nos sociétés » (Rieffel, 2014, p. 15), remet en cause ces distinctions. Ainsi on remarque la création de nombreuses archives en ligne produites aussi bien par des amateurs que par des professionnels non archivistes (Besson, 2014). Dans le même temps, on note une multiplication des usages artistiques des archives (Bénichou, 2014). De manière générale, la création de sites documentaires et d'archives en ligne conduit à ce que les internautes puissent consulter eux-mêmes des sources premières en version numérique (Theimer, 2014). La distinction entre domaines de la trace et de la représentation est ainsi contestée. L'archive, entendue cette fois comme institution, est, en effet, donnée à voir comme une production culturelle située dans le temps. Elle est, de manière comparable (et non équivalente) au film, une représentation de l'histoire, soit le résultat d'une gestion de la présence du passé dans l'espace public (Méchoulan dir., 2011). Il résulte de l'ensemble de ces éléments une sorte de flou notionnel autour d'une série culturelle, soit d'un «sous-systèmes ou unités de 
signification composant le plus grand ensemble » qui correspond à ce que le numérique fait au cinéma (Gaudreault, 2007, p. 114).

3 Lancé en fin mars 2014, le site du projet Man on Bridge s'inscrit dans la tendance susmentionnée. Il en est un symptôme (Rousso, 1990). En effet, il se donne à consulter, à la fois comme un fonds d'archives en ligne, un webdocumentaire et un documentaire interactif. Dans le cadre de cet article, ces trois catégories seront tour à tour utilisées pour analyser ce projet qui est aussi composé d'un film documentaire, d'un livre et d'une exposition. Elles seront également contestées, en s'appuyant sur les catégories de collection et de projet transmédia. Enfin, un modèle interprétatif alternatif basé sur les dimensions juridique et économique sera proposé. Il conduira à faire émerger la notion de base de données et de matrice documentaire. Ainsi, à travers une étude de cas clairement circonscrite, l'enjeu de ce texte est une réflexion méthodologique concernant le mode d'interprétation le plus adapté à ce type de format, qui entre cinéma et archive, conduit à repenser notre rapport au passé à l'époque du numérique.

\section{Entre fonds d'archives et documentaire interactif}

4 Le premier modèle interprétatif adopté est d'ordre sémiopragmatique (Odin, 2011). Il s'agit de porter une attention tout à la fois à la forme prise par l'interface, ainsi qu'à la manière dont il en est fait usage par les internautes.

\section{Les multiples images d'Arthur Fields}

5 Le site du projet Man on Bridge adopte le format d'un fonds d'archives ${ }^{1}$, car il donne accès à un corpus d'images prises par un photographe irlandais, Arthur Fields (1901-1994). Il s'agit dans cette partie de s'intéresser à la forme de cette interface. Sur la page d'accueil, notons qu'une dizaine de ces photographies s'affichent par défaut. En noir et blanc, au format vertical, celles-ci représentent une famille, un couple, un homme pressé, une petite fille et un groupe d'amis (cf. figure 1). Elles sont accompagnées d'entretiens filmés pour le site par le réalisateur irlandais Ciaran Deeney. Dans la première de ces vidéos les trois fils de Fields expliquent qu'il était un photographe de rue ayant travaillé quotidiennement sur le pont O'Connell à Dublin pendant plus de cinquante ans (1932-1988). Son métier consistait à attirer l'attention des passants, puis à prendre leur portrait. Munis d'un ticket, ces derniers étaient ensuite invités à récupérer un tirage contre le paiement d'une somme relativement modeste.

Figure 1. Capture d'écran des images apparaissant sur la page d'accueil du site Man on Bridge.

6 Si on fait défiler vers le bas (scrolling ou scroll down) l'unique colonne qui compose l'interface (ainsi adaptée à une consultation sur tablette ou téléphone intelligent ${ }^{2}$ ), d'autres photographies s'affichent par groupe de dix. Ce geste peut être répété à de nombreuses reprises, ce qui rend compte du fait qu'un large corpus d'images a été collecté. Deeney avance le nombre de 5000 photographies sur les 182500 prises par Fields ${ }^{3}$. Les onglets en haut de page permettent eux d'afficher directement toutes les vidéos du site, les photographies par période de prise de vue (l'unité est la dizaine d'années) ou par thèmes (ils sont au nombre de 36, dont, par exemple, chapeaux, journaux, trams, valises ou encore parapluies). À tout moment, il est aussi possible de cliquer sur une photographie, celle-ci s'intègre alors à une visionneuse qui s'ouvre en overlay. Elle est 
accompagnée de la mention d'une année de prise de vue ainsi que d'une légende portant sur le contenu de l'image et/ou sur la biographie des personnes représentées. Chaque légende (ou presque) est signée par une personne différente. La présence de l'ensemble de ces éléments renforce l'impression d'être face à l'archive vernaculaire d'un photographe.

\section{Un parcours adapté au web}

7 Si le principal attrait esthétique de l'interface réside dans l'affichage par grappes d'images, l'interface peut également être considérée comme étant un webdocumentaire, car la narration est assurée via des capsules audiovisuelles. Le terme webdocumentaire est ici utilisé pour décrire un format adapté à une diffusion en ligne (principe d'interactivité, éléments audiovisuels fragmentés, etc.), par distinction du film documentaire qui est, lui, pensé pour une projection en salle ou une retransmission télévisée (Di Crosta, 2009). L'idée principale, comme l'écrit William Urrichio est que les internautes "prennent en main la construction de leur propre texte à partir des matériaux et du contexte qui leur sont donnés » $(2014$, p. 65). Ils construisent leur propre parcours. Au nombre de neuf, pour un peu plus de vingt minutes, les vidéos mises en ligne donnent la parole à onze personnes aux statuts différents. Il y a les fils d'Arthur Fields (Philip, Bernard et David Fields), deux collectionneurs de photographies (Brian Quinn et Garry O'Neill), deux historiennes (Orla Fitzpatrick qui travaille sur la photographie et Ruth Grifin, spécialiste de la mode), un photographe professionnel (David Davison), un vendeur de photographies qui travaillait non loin du pont O'Connell (Brian Whelan) et deux personnes qui ont participé à retrouver des éléments du fonds (David Denny a communiqué les nombreuses photographies de son père et Martin Mooney a acquis lors d'une vente aux enchères un des appareils de Fields). Il ressort de ces capsules, dont le montage est basé sur le principe d'une alternance entre des photographies issues du corpus et d'entretiens filmés face caméra devant un fond neutre, que l'intérêt du projet n'est pas directement lié à la qualité esthétique des clichés ${ }^{4}$. Ainsi, l'interface étudiée ne vise pas à faire accéder les photographies d'Arthur Fields au monde de l'art (Heinich, 2001), comme cela a pu être le cas pour différents photographes de rue ces dernières années ${ }^{5}$. Il s'agit plus de le présenter comme un témoin privilégié de son temps. David Fields explique ainsi que ces images constituent une partie de l'histoire et de la culture de Dublin (capsule 1). Martin Mooney (capsule 2) et David Denny (capsule 5) indiquent qu'elles rendent possible un retour dans le temps (step back in time). Brian Quinn et David Davison insistent, eux, sur le fait que c'est la longévité de la présence d'Arthur Fields sur le pont O'Connell qui fait sa spécificité vis-à-vis des autres photographes présents sur ce lieu touristique (capsules 3 et 7). Les deux historiennes mettent, elles, en valeur l'intérêt du corpus pour une histoire de l'habillement quotidien des Irlandais (capsules 4) et pour comprendre l'évolution de la culture des jeunes dublinois (capsule 6). Brian Quinn note également qu'elles donnent accès aux inscriptions reportées sur les façades de magasins qui n'existent plus aujourd'hui'. Ainsi, les photographies sont présentées comme étant autant de « documents sur l'évolution de la société » (Davison, capsule 8). L'optique adoptée est donc à la croisée d'une histoire culturelle d'une pratique photographique singulière et d'une sociologie visuelle portant sur un demi-siècle de la vie de Dublin (Chauvin et Reix dir., 2015). 


\section{Une collecte d'images privées}

8 Cette présentation de l'interface comme archive, puis comme webdocumentaire est incomplète, car celle-ci relève aussi du format documentaire interactif. Cette nouvelle expression correspond non seulement à un format adapté à une diffusion en ligne, mais aussi à la prise en compte de l'usager comme co-constructeur du contenu de la plateforme. Elle se situe, au minimum, au niveau de l'intégration des bases du web 2.0 (Cardon, 2009). Dans ce cas, on note que le partage des photographies et de leurs légendes, ainsi que des capsules audiovisuelles sur les principaux réseaux sociaux (Twitter, Facebook et Pinterest) est encouragé (icônes présents dans la visionneuse). Cependant, l'originalité de la forme ne réside pas à ce niveau-là. Il s'agit de la possibilité offerte à tous les internautes d'ajouter leurs images prises par Fields à l'archive en ligne.

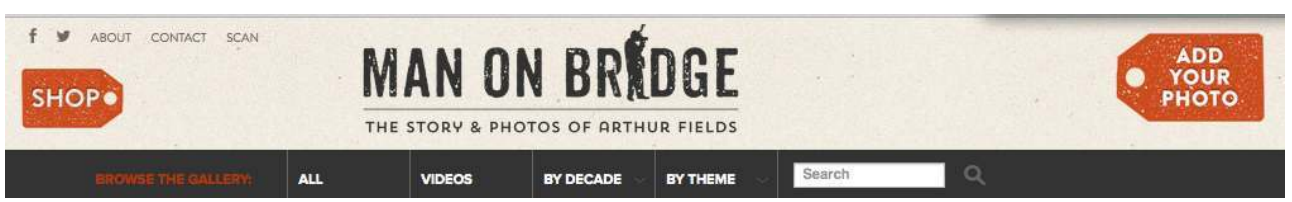

Figure 2. Capture d'écran du bandeau apparaissant sur le site Man on Bridge.

9 Cela est rendu possible via un formulaire dont l'accès est facilité par un bouton sur le bandeau principal de la page accueil (cf. figure 2). Il est alors demandé d'associer à l'image une légende et une année de prise de vue, ainsi que d'indiquer leur nom (et leur adresse email). Ces informations correspondent aux métadonnées liées à chacune des images quand elles sont ouvertes dans la visionneuse. Le projet Man on Bridge est donc un cas d'indexation sociale (crowdsourcing ${ }^{7}$ ). L'objectif est ici la constitution d'une collection d'images par un appel à la bonne volonté de bénévoles. Ces derniers apportent, à la fois la trace du passé qu'ils ont conservée, la photographie, et ils la documentent en ajoutant des informations qui sont communiquées via un formulaire. À ce titre, il s'agit bien d'une archive en ligne. L'utilité de ce projet collaboratif est liée au fait, qu'Arthur Fields n'a pas conservé les négatifs des photographies qu'ils prenaient et que les tirages étaient vendus. Les images prises par lui existent donc, mais elles étaient disséminées, principalement chez ceux dont le photographe a pris l'image entre 1932 et 1988. Ces documents sont intégrés à une interface dynamique également constituée de capsules audiovisuelles, ce qui fait que l'interface proposée est également un documentaire interactif en constante évolution. Le flou notionnel évoqué en introduction cède ici le pas à une triple désignation de la forme (archive en ligne, webdocumentaire et documentaire interactif).

\section{Une forme inclassable?}

Une fois ce triple statut posé, il est à présent possible d'en contester la légitimité. Cela nécessite de passer d'une analyse sémiopragmatique à une approche génétique (analyse du processus de réalisation) et à une réinscription de la plateforme dans un projet transmédia. 


\section{De la désignation comme fonds d'archives à celle de collection}

11 L'assimilation du site Man on Bridge à une archive en ligne peut être contestée car s'il y a bien indexation sociale, celle-ci ne respecte pas les normes de base de l'archivistique. Cela est particulièrement criant en ce qui concerne le choix des données associées aux images numérisées. Celles-ci se limitent à une date, une description de l'image et à l'identification du dépositaire du document. Ainsi, aucune information n'est transmise à l'usager de l'interface au sujet de la matérialité de l'image. Par exemple, il ne connaît pas les dimensions de l'image ou le type de papier. Il ne sait pas non plus si l'image est tirée d'une série et/ou si le dépositaire a numérisé d'autres documents. De plus, le lien entre le document physique et la version numérisée n'est pas assuré. L'usager de la plateforme ne sait pas s'il a été conservé et, si oui, dans quelles conditions ${ }^{8}$. Enfin, l'absence de motsclefs interdit à l'usager toute recherche avec des catégories autres que celles proposées par l'interface (36 susmentionnées). Il n'est ainsi pas possible de croiser une date ou une décennie, avec une catégorie, afin de mener une recherche plus fine. Au-delà des métadonnées, notons également que le dos de l'image n'a pas été numérisé. La conservation des deux faces de l'image est pourtant l'un des acquis de l'archivistique contemporaine dans le domaine de la photographie (About et Chéroux, 2001). Le dos l'image permet souvent d'obtenir des informations sur les conditions de réalisation de celle-ci (et ainsi de dépasser une analyse qui se limite à l'analyse du contenu de l'image). Enfin, la véracité des informations communiquées n'a pas été vérifiée. La datation et la description de l'image sont du seul fait du dépositaire. Cela conduit à une très grande diversité de descriptions (absence de normalisation), certaines ne comportant que quelques mots, d'autres revenant longuement sur la biographie des personnes photographiées. Plus grave d'un point de vue archivistique, il n'est pas certain que toutes les images partagées soient d'Arthur Fields. Il se peut très bien que des photographies prises par David Fields (le frère d'Arthur), Con Keane, Dany Delahunty, Harry Cowan, Max Coleman, John Quinn, soit quelques-uns des autres photographes travaillant sur le pont O'Connell, aient été intégrées ${ }^{9}$. Ce dernier point conduit à ce que pour la suite de cet article, on préfère utiliser le terme de collection ${ }^{10}$. Dans tous les cas, ces manques conduisent à douter du fait que l'objectif poursuivit par l'équipe en charge de la réalisation du site soit véritablement la constitution d'une archive en ligne.

\section{Du documentaire interactif au storytelling transmédia}

12 Le statut de documentaire interactif est lui moins contestable que celui d'archive. Il est cependant nécessaire de replacer la forme étudiée au sein d'un projet plus large, qui peut être considéré comme relevant du storytelling transmédia. Rappelons que ce terme est utilisé par Henry Jenkins pour définir une production culturelle qui «se déploie sur de multiples plateformes médiatiques, chaque texte nouveau apportant à l'ensemble une contribution différente et précieuse»(2014, p.119). Man on Bridge entre dans cette catégorie, car en plus d'un documentaire interactif, il se compose aussi d'un film documentaire de 50 minutes, d'une exposition, d'un livre, ainsi que de comptes actifs sur différents réseaux sociaux ${ }^{11}$. Le modèle du transmédia est ici adapté, car il n'y a pas une production culturelle centrale, dont les autres seraient des produits dérivés, mais bien une multitude de projets qui entrent en synergie. 


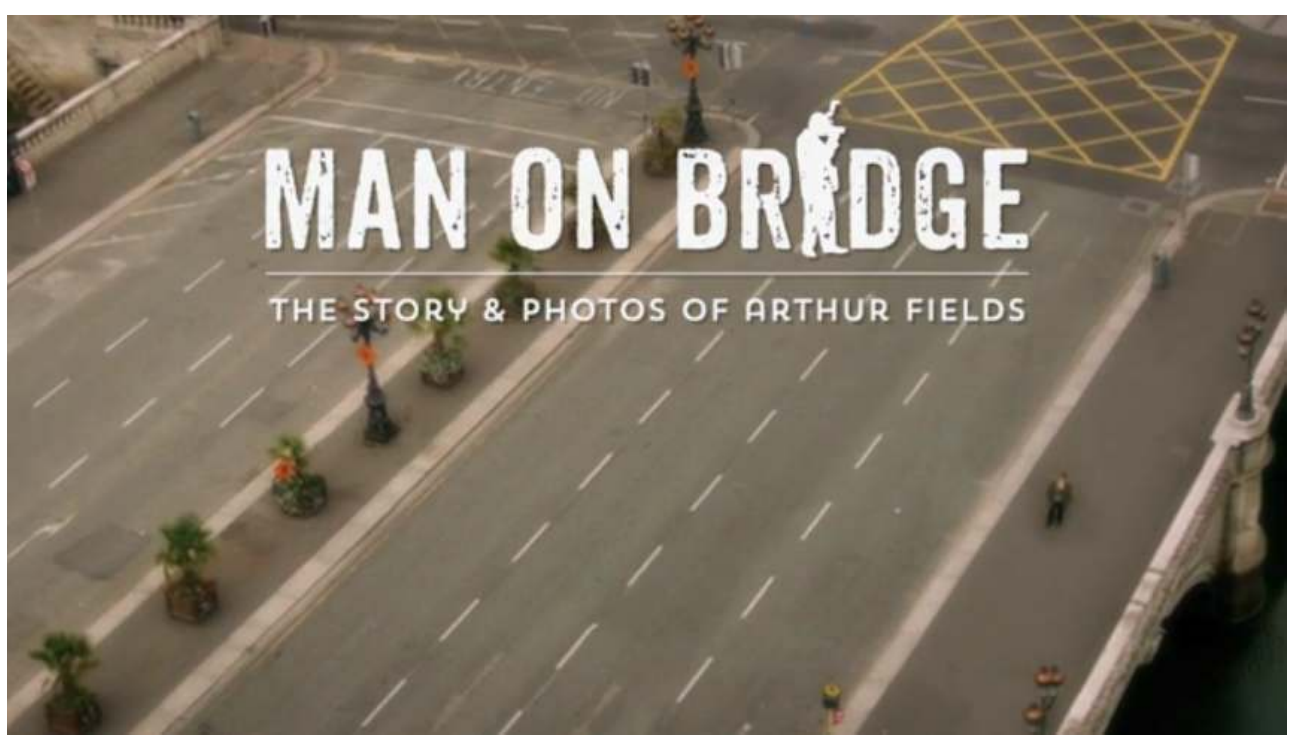

Figure 3. Capture d'écran de l'ouverture du film documentaire Man on Bridge.

13 Cela est lié à la genèse de Man on Bridge. Au départ, Deeney avait développé un modèle de storytelling transmédia portant sur un tout autre sujet. Il s'agissait d'une coproduction soutenue par l'Office National du Film canadien (ONF). Ce projet basé sur la collecte d'images documentées par des bénévoles n'ayant pas vu le jour (Caribbean Island, non daté ${ }^{12}$ ), le réalisateur a cherché un autre sujet d'expérimentation (Deeney et Molumby, 2014). Le projet est donc issu d'une réflexion sur les transformations actuelles du documentaire à l'époque du numérique. À la même époque, la famille d'Arthur Fields cherchait à lui rendre hommage ${ }^{13}$. Il y a donc eu un effet d'opportunité. Un modèle économique a alors été trouvé pour le site grâce à l'obtention d'une bourse de cinquante mille euros (Arthur Guinness Project, septembre 2013). Le film documentaire a lui été rendu possible par le soutien de la chaîne de télévision publique irlandaise RTE One (mars 2014). Il a été diffusé le 28 décembre 2014. En parallèle, une exposition a été mise en place par la très reconnue Gallery of Photography de Dublin (novembre 2014 - janvier 2015 ${ }^{14}$ ) et un livre publié, le soutien du conseil de la ville venant s'ajouter aux autres.

Retenons ici que la définition proposée par Jenkins a été suivie à lettre, puisque chaque média a été utilisé pour représenter différents aspects du projet (il n'y a pas eu déclinaison d'un même récit). En effet, si le site a surtout servi à présenter un projet d'histoire culturelle du visuel à Dublin (voir supra), le film est lui centré sur les multiples facettes du personnage Arthur Fields ${ }^{15}$. Les entretiens montés en ligne n'ont pas tous été réutilisés. La parole des chercheurs et des collectionneurs n'a pas été remobilisée, c'est, avant tout, la famille qui a été invitée à s'exprimer. L'accent a aussi été mis sur les personnes célèbres photographiées sur le pont O'Connell (George Harrison, Margaret Rutherford et Brendan Behan, notamment). L'exposition elle, a plus mis l'accent sur le caractère artistique de la pratique d'Arthur Fields. Ainsi, cette dimension qui n'était pas au centre du documentaire interactif, a trouvé un espace pour s'exprimer. L'ouvrage constitue lui une remédiation des images téléchargées en ligne. À la différence du site, il donne accès à celles-ci dans un format de consultation confortable. De nombreuses photographies sont reproduites en pleines pages (15 centimètres de largeur), alors que leur faible résolution ( $72 \mathrm{dpi}$ ) et dimension (environ 350 pixels de large) interdisaient tout tirage de qualité depuis le site. Enfin, sur les réseaux sociaux, la diffusion quotidienne 
d'images issues de la collection créée un lien continu entre l'équipe du projet et les usagers $^{16}$.

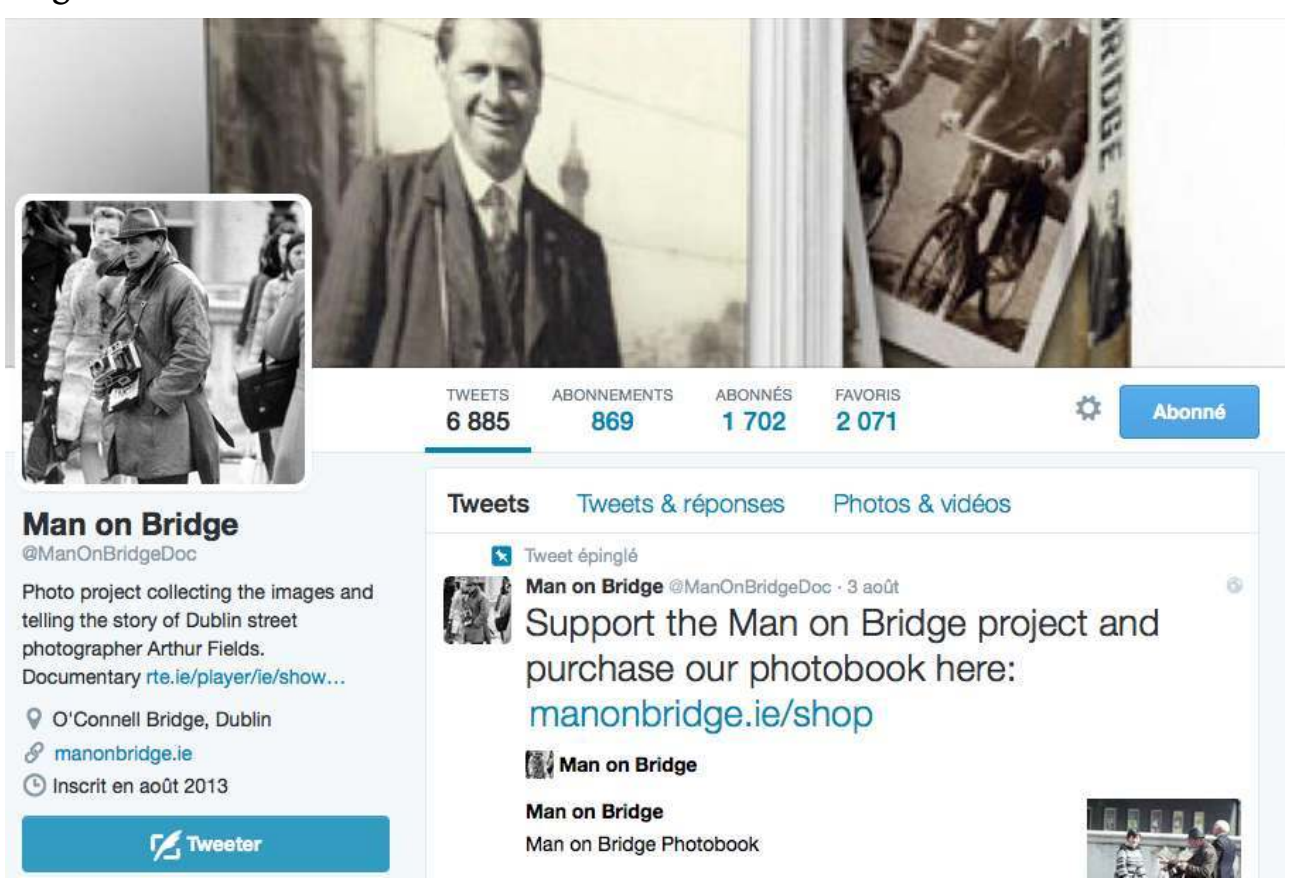

Figure 4. Capture d'écran du compte Twitter du projet Man on Bridge. Celui-ci met en avant la publication du livre éponyme.

\section{L'émergence d'un modèle alternatif : la base de données}

15 Le point commun à l'ensemble de ces productions culturelles est l'usage du corpus de photographies attribuées à Arthur Fields et collectées sur le site. Celles-ci sont constamment délocalisées, puis relocalisées dans un nouvel environnement médiatique (Treleani, 2014, p. 113-115), peu importe que celui-ci soit numérique ou non. Ce constat conduit à proposer une autre interprétation de la plateforme. Celle-ci peut être considérée comme une matrice documentaire ayant permis la conception des autres productions culturelles. Cette interprétation est soutenue par le fait que le dépôt d'une photographie sur la plateforme est associé à une cession des droits sur l'image. Les termes et conditions accessibles en ligne débutent ainsi :

By submitting your photographs you agree to grant El Zorrero Films (the 'producer') a perpetual, royalty-free, irrevocable, non-exclusive, sub-licensable right and license to use, reproduce, modify, adapt, publish, create derivative works from, distribute, make available to the public, and exercise all copyright and publicity rights with respect to your photograph worldwide and/or to incorporate your photograph in other works and publications in any form, media or technology now known or later developed for the full term of any rights that may exist in your photograph ${ }^{17}$.

En somme le dépositaire reste en possession de la photographie, mais l'usage de celle-ci dans l'espace public est soumis à un Copyright qui est détenu par le producteur du projet (El Zorrero). Cela explique pourquoi la mention suivante est reportée à la fin de l'ouvrage Man on the Bridge :

Ciaran Deeney has asserted his moral right to be identified as the author of this work in accordance with the Irish Copyright and Related Rights Act 2000. 
19 All rights reserved. The material in this publication is protected by copyright law. Except as may be permitted by law, no port of the material may be reproduced (including by storage in a retrieval systeme) or transmitted in any form or by any means, adapted, rented or lent without the written permission of the copyright owners ${ }^{18}$.

Pour résumer, les photographies sont gracieusement numérisées, puis téléchargées sur le site par le dépositaire, ce qui équivaut à une cession de droits envers le producteur. Le réalisateur peut ensuite utiliser ces images dans des productions commerciales telles qu'un documentaire télévisé ou un livre vendu au grand public. Cela correspond à un processus de transfert de propriété, qui permet la monétisation des photographies par El Zorrero. Celui-ci explique notamment la faiblesse de la résolution des images mises en ligne sur le site (cf. supra). Ainsi, à la différence d'une licence de type Creative Commons ${ }^{19}$, l'usager de la plateforme ne peut pas réutiliser les images ou les intégrer à d'autres productions, que celles-ci soient amateurs ou professionnelles. Si on suit cette interprétation du site, celui-ci correspond moins à une archive ou à une collection, qu'à la constitution d'une base de donnée ${ }^{20}$ au service de la réalisation d'un ensemble de productions culturelles. Les trente-six thèmes mentionnés sur l'onglet de la page d'accueil du site sont l'équivalent des entrées qui sont proposées dans les bases créées lors des recherches iconographiques menées en amont de la réalisation d'un documentaire historique ${ }^{21}$. Cela peut constituer un facteur explicatif de l'empressement ressenti par l'équipe d'El Zorerro à mettre en place des journées de numérisation dans différentes bibliothèques de Dublin ${ }^{22}$, ainsi qu'à passer un partenariat avec plusieurs boutiques de numérisation (Cf. The Pictorium ${ }^{23}$ ). L'objectif du site est de retrouver un maximum de photographies afin de pouvoir mener d'autres projets. Ce processus a été un succès d'un point de vue quantitatif puisque le nombre d'images collectées est passé de 1800 en avril 2014 (Finn, 2014) à près de 5000 fin 2014 (Deeney et Molumby, 2014).

\section{Le terme d'archive comme argument rhétorique}

21 Cet éloignement vis-à-vis de la catégorie archive conduit à s'interroger sur l'usage de ce terme. Une analyse du discours d'accompagnement du projet dans l'espace public est alors nécessaire afin de comprendre la prégnance de cette catégorie. Il en ressort que cet usage est le fait de l'équipe. Par exemple, sur le site de la Gallery of Photography, l'exposition est présentée ainsi :

This impressive, must-see installation includes a massive billboard-type display of every image so far contributed to the Man on Bridge archive - giving visitors the chance to see in one sweep over 3,400 images spanning 50 years of Dublin street life and style (op. cit.) ${ }^{24}$

De même, le producteur et l'entreprise ayant conçu le site expliquent :

The goal of the project is to gather these photos from personal collections and create an alternative photographic archive of Dublin city, one that reveals how we lived, how the city and its people changed over fifty years (op. Cit.) ${ }^{25}$.

Ces propos sont repris, dès 2013, par les partenaires du projet ${ }^{26}$ et par les médias qui s'intéressent à celui- $\mathrm{ci}^{27}$. Ainsi, dans The Observer, le magazine dominical associé à The Guardian, Sean O'Hagan écrit : 

une réévaluation de la qualité esthétique des photographies d'Arthur Fields, ni à identifier des aspects particulièrement originaux dans la forme prise par l'interface. Ce constat a mené à considérer que l'intérêt du site Man on Bridge se situe moins au niveau formel, qu'au niveau des fonctionnalités et des usages de l'interface de la part des internautes. La catégorie générique webdocumentaire (permettant d'insister sur l'adaptation de la forme au média web) a alors été abandonnée au profit de celles de documentaire interactif (soulignant plus le rôle des usagers) et de fonds d'archives en ligne (car c'est l'ensemble des images prises par un individu qui sont mises en ligne). Une attention toute particulière portée à la catégorisation du site a conduit, dans la seconde partie de cet article, à remettre en cause ces trois désignations. Le modèle sémiopragmatique a alors cédé la place à une démarche de type génétique (focaliser sur le processus de réalisation) et à une réinscription du site dans un projet plus large. Ainsi, les limites identifiées en termes de respect des normes archivistiques ont conduit à l'usage du terme collection. Les liens établis avec la réalisation d'un film documentaire, la conception d'une exposition et l'édition d'un ouvrage, ont eux mené à percevoir le site comme un élément d'un storytelling transmédia. Cette nouvelle remise en cause a conduit à dégager un modèle interprétatif alternatif. Celui-ci repose sur l'identification d'un procédé juridique permettant la monétisation des images. Il est alors proposé de concevoir le site Man on Bridge comme une base de données au service de la réalisation des autres facettes du projet. Elle est, à ce titre, une sorte de matrice documentaire. Il s'agit, au-delà de ce cas, d'une des fonctions possibles d'un documentaire interactif, dans un projet plus développé. 
Pour conclure notons que ces sept désignations (webdocumentaire, documentaire interactif, fonds d'archives en ligne, collection, élément d'un projet transmédia, base de données et matrice documentaire) sont moins à considérer comme étant exclusives les unes des autres que comme étant complémentaires. Le propre d'une série culturelle est, en effet, le maintien d'un certain degré d'hétérogénéité entre ces composantes. Il ne s'agit pas encore d'un média institutionnalisé autours d'un modèle à la fois économique, technique et socio-culturel (Besson, 2014). La complexité des projets mis en place à l'époque du numérique réside dans le fait qu'ils reposent sur une forme d'hybridation entre ces types qui étaient préalablement plus clairement distinguables. Au terme de cette réflexion méthodologique, arrimée à une étude de cas, il reste à insister sur le fait que ce rapprochement ne correspond pas à l'identification d'une confusion d'ordre conceptuel. Dans le cadre de cet article, nous avons tenter de démontrer qu'il est toujours aussi important, d'articuler, sans les confondre, ces différents termes. C'est par la conjonction de ces points de vue, qu'il a été progressivement possible de faire émerger la spécificité d'une série culturelle, qui renouvelle actuellement les rapports entre archives et cinéma, ainsi que plus largement la présence du passé dans le présent.

\section{BIBLIOGRAPHY}

Bibliographie :

Agnew, Agnew Roisin, « Arthur Fields: Man on Bridge », Totally Dublin, 2014.

Anonyme, « The Photographer on the Bridge: 50 Years of Undiscovered Street Snaps ", MessyNessy, 5 août 2013.

Anonyme, « Garbage Island, the interactive documentary, nears.... », Blog de El Zorrero, 13 janvier 2014.

Anonyme, « Arthur Fields putting life in focus », Irish Examiner, 15 décembre 2014.

Bénichou, Anne, Un imaginaire institutionnel. Musées, collections et archives d'artistes, Paris, L'Harmattan, 2014.

Besson, Rémy, « Prolégomènes pour une définition de l'intermédialité à l'époque contemporaine ", rapport de recherche publié sur l'archive ouverte HAL-UTM, juillet 2014.

Besson, Rémy, « Regard intermédial sur le devenir numérique des archives ", Intermédialités, $\mathrm{n}^{\circ}$ 23, printemps 2014.

Chauvin, Pierre-Marie et Fabien Reix (dir.), «L’Année sociologique », vol. 65, $\mathrm{n}^{\circ} 1$ : Sociologie visuelles, 2015.

Deeney, Ciaran, et Christina Finn (entretien avec), « Do you remember photographer Arthur Fields of O'Connell Bridge? », The Journal.ie, 18 août 2013.

Deeney, Ciaran, et Christina Finn et Deirdre Molumby (entretien avec), « Multi-Platform Doc 'Man on Bridge' Airs this Christmas », The Irish Film \& Television Network, 22 décembre 2014. 
Deeney, Ciaran et David Clarke, Man on Bridge. The Photos of Arthur Fields, Dublin, The Collins Press, 2014.

Delage, Christian et Vincent Guigueno, L'Historien et le film, Paris, Gallimard, 2004.

Di Crosta, Marida, Entre cinéma et jeux vidéo : l'interface-film, Bruxelles, De Boeck, 2009.

Finn, Christina, «Photo project collects 1,800 of your photos taken over 50 years on O'Connell Street », The Journal.ie, 6 avril 2014.

Gaudreault, André, Cinéma et attraction. Pour une nouvelle histoire du cinématographe, Paris, CNRS Éditions, 2007.

Heinich, Nathalie, La Sociologie de l'art, Paris, La Découverte, 2001.

Jenkins, Henry, La Culture de la convergence. Des médias au transmédia, Paris, Armand Colin, 2014 (1ère éd. en anglais, 2006).

Méchoulan, Éric (dir.), Intermédialités, n 18 : Archiver/Archiving, 2011.

Morrissey, Priska, Historiens et cinéastes : rencontre de deux écritures, Paris, L'Harmattan, 2004.

Odin, Roger, Les espaces de communication. Introduction à la sémio-pragmatique, Grenoble, Presses universitaires de Grenoble, 2011.

Rieffel, Rémy, Révolution numérique, révolution culturelle?, Paris, Gallimard, 2014.

Rousso, Henry, Le syndrome de Vichy, Paris, Seuil, 1990 (2ème éd.).

Smyth, Damian, « Arthur Fields: The Man on the Bridge », The Digital Feed, 20 septembre 2014.

Theimer, Kate, « The Future of Archives is Participatory: Archives as Platform, or A New Mission for Archives ", ArchivesNext, 3 avril 2014 [en ligne].

Treleani, Mattéo, Mémoires audiovisuelles. Les archives en ligne ont-elles un sens ?, Montréal, Presses de l'Université de Montréal, 2014.

Uricchio, William, « Repenser le documentaire social », dans Laurence Allard et all. (dir.), Téléphone mobile et création, Paris, Armand Colin, 2014, p. 61-79.

Veray, Laurent, Les images d'archives face à l'histoire. De la conservation à la création, Paris, Scérén/ CNDP, 2011.

\section{NOTES}

1. Rappelons la définition de cette expression par l'Association des professionnels de l'information et de la documentation (ADBS), « Ensemble des documents de toute nature qu'une personne physique ou morale a automatiquement produits ou reçus dans l'exercice de ses activités, rassemblés et organisés en conséquence de celles-ci, et conservés en vue d'une utilisation éventuelle. " [en ligne]. Sauf mention contraire, les pages citées ci-dessous ont été consultées le 8 mai 2015.

2. Cet aspect est le principal point mis en avant par l'entreprise qui a développé le site. Cf. visuel sur la page de Pixel Design, URL : http://www.pixeldesign.ie/work/man-on-bridge

3. L'estimation du nombre total de photographies est basée sur le fait qu'il a pris au moins dix photographies quotidiennement (365 jours par an) pendant cinquante ans (Deeney et Molumby, 2014). 
4. Cela est assumé par le réalisateur qui explique, par exemple, «that Arthur's photos were never magnificent, but they are "natural" and capture a sense of each era » (Deeney et Finn, 2013).

5. Le film documentaire Finding Vivian Maier (John Maloof and Charlie Siskel, 2013) est représentatif de cette tendance. Il est aussi possible de penser à Everybody Street (Cheryl Dunn, 2013).

6. L'historien Donal Fallon revient sur cet aspect dans le cadre de l'émission The History Show sur RTE Radio 1, 30 novembre 2014 [en ligne].

7. Il s'agit d'une pratique archivistique revenant à intégrer des informations communiquées par ceux qui ont eu usage des artefacts archivés.

8. Les images sont, en fait, conservées dans les bibliothèques de Dublin, lire Deeney et Finn, 2014.

9. Cet aspect est explicitement reconnu dans l'introduction à l'ouvrage Man on Bridge. Après avoir cité les noms susmentionnés, l'auteur indique « Undoubtedly, some of their photos spill into this book» $(2014$, p. 4).

10. Rappelons qu'une collection correspond à un «regroupement volontaire de documents, d'objets, d'informations de provenances diverses, rassemblés en raison de la similitude d'un ou de plusieurs de leurs caractères (contrairement au fonds d'archives qui est constitué organiquement) », ADBS [en ligne].

11. Une projection de photographies est également organisée dans le cadre de la Culture Night à Dublin (équivalent de la Nuit blanche parisienne) le 19 septembre 2014 [en ligne].

12. Lire : " Garbage Island, the interactive documentary, nears.... », El Zorrero, 13 janvier 2014 [en ligne].

13. Cela s'est notamment traduit par la mise en ligne d'un montage audiovisuel amateur sur YouTube en 2008. Comme cela est rappelé dans un article de 2013, David Fields avait également créé une page internet permettant de lui envoyée des photographies prises par son père, URL: http://www.abovethebest.ie/manonbridge/ Pour consulter l'article faisant le lien avec cette page, lire : anonyme, « The Photographer on the Bridge » (2013).

14. Site de l'exposition, URL : http://www.galleryofphotography.ie/man-bridge/

15. Le film n'est pas hagiographique. Il dépeint un homme tourmenté, parfois dur envers sa femme et ses enfants, dans tous les cas obsédé par son travail. Pour une présentation plus complète lire : « Arthur Fields putting life in focus » (2014).

16. La présence de l'équipe est remarquable, puisqu'ils répondent constamment aux sollicitations et qu'ils reprennent régulièrement les messages partagés par les usagers du site Man on Bridge et/ou de ces réseaux. D'un point de vue quantitatif, on note 1620 abonnés au compte Twitter et 9386 inscrits sur la page Facebook. (Pages consultées 11 mai 2015).

17. «En soumettant vos photographies, vous acceptez d'accorder à El Zorrero Films (le « producteur »), le droit perpétuel, libre de redevance, irrévocable, non-exclusif et une licence pour utiliser, reproduire, modifier, adapter, publier, créer des œuvres dérivés, distribuer, mettre à la disposition du public et d'exercer tous les droits d'auteurs et les droits de publicité à l'égard de votre photographie dans le monde entier et /ou d'intégrer votre photographie dans d'autres œuvres et publications de toute forme, média ou technologie connue ou éventuellement développée pour le plein terme de tous droits qui peuvent exister concernant vos photographies ». 
18. «Ciaran Deeney a fait valoir son droit moral d'être identifié comme l'auteur de ce travail en conformité avec la Loi irlandaise sur les droits d'auteur et les droits connexes de 2000.

Tous droits réservés. Le contenu de cette publication est protégé par la Loi sur les droits d'auteur. Sauf dans la mesure permise par la Loi, aucune partie du matériel ne peut être reproduite (y compris par le stockage dans un système de récupération) ou transmise sous quelque forme ou par quelques moyens que ce soit, adaptée, louée ou prêtée sans la permission écrite des titulaires des droits d'auteur ».

19. «Creative Commons (CC) est une organisation à but non lucratif dont le but est de proposer une solution alternative légale aux personnes souhaitant libérer leurs œuvres des droits de propriété intellectuelle standards de leur pays, jugés trop restrictifs. », Page Wikipédia [en ligne].

20. Cette expression est utilisée sur la page de El Zorrero, "This growing database of user-submitted photos will be integrated into our online story of Arthur on a rolling basis as photos are submitted. » [en ligne]

21. Ce type de pratique a notamment été présenté par l'équipe de la série Apocalypse, A. Dauer (assistant-réalisateur), M. Rougeron (monteuse, 3ème épisode) et M.Arghib (stagiaire documentaliste), lors d'une séance de l'atelier Les enjeux de la narrativité dans le cinéma dit documentaire (EHESS, 5 mai 2010), coordonné par l'auteur de cet article.

22. La liste des lieux assurant la numérisation est accessible sur le site, URL: http:// www.manonbridge.ie/scan-locations

23. URL: http://pictorium.ie/arthur-fields-man-on-bridge-photo-archive-project/ Consulté le 10 août 2015.

24. Cette impressionnante installation, à découvrir absolument, comprend un énorme écran de type panneau d'affichage réunissant toutes les images ayant contribué jusqu'à présent aux archives de Man on Bridge - donnant aux visiteurs la chance de voir d'un seul coup plus de 3400 images couvrant 50 ans de vie urbaine à Dublin.

25. L'objectif du projet est de rassembler ces photos provenant de collections personnelles et de créer une archive photographique alternative de la ville de Dublin qui révèle comment nous vivions, comment la ville et ses habitants ont changé depuis cinquante ans.

26. Par exemple, la citation ci-dessus figure textuellement sur le site The Séamus Ennis Arts Centre lorsqu'il annonce une projection du film pour le 11 janvier 2015 [en ligne].

27. Par exemple, la citation ci-dessus figure textuellement sur la base de données que MIT (Boston) consacre aux documentaires interactifs, URL : http://docubase.mit.edu/project/ man-on-bridge/ Page consultée le 8 mai 2015.

28. Sachant qu'il doit y avoir des milliers de ces clichés enfouis dans les placards et les tiroirs de toute l'Irlande, les cinéastes visent à souligner le travail des photographes amateurs en encourageant les gens à soumettre leurs photos pour une archive en ligne. Cette phrase se retrouve également sous la plume de Damian Smyth (2014).

29. (...) les cinéastes ont encouragé les gens à soumettre leurs photographies dans une archive en ligne.

30. Sur le site culturel Totally Dublin, Roisin Agnew indique, "Ciaran Deeney talks about 'the responsibility to do something good with other people's photographs and memories.' (...) He wanted a broad open-access platform » (2014). 


\section{ABSTRACTS}

The digital project Man on Bridge gives access to a corpus of photographs taken by Arthur Field (1901-1994). This Irish photographer took pictures every day on the O'Connell Bridge (Dublin) during more than fifty years (1932-1988), but he kept no negative. This research paper leads to understand that the main interest of the project is neither based on theaesthetic quality of the photos, nor in the formal originality of the website created in 2014 by Ciaran Deeney. The real originality of Man on Bridge lies more in the particular relationship created with the users. Indeed, the project does not only tell Arthur Field story but also aims to collect his photos preserved by the Dubliners. This observation leads to rethink the basic concepts used to interpret this kind of projects: Web Documentary, Interactive Documentary, Online Archive, Transmediastorytelling or Digital Database. To sum up, this case study is a methodological reflexion dealing with the adaptation of the cinematic storytelling to the advent of the Digital Age.

Le site du projet Man on Bridge donne accès à un corpus d'environ 5000 images prises par un photographe irlandais, Arthur Fields (1901-1994). Celui-ci a travaillé comme photographe de rue sur le pont O'Connell à Dublin pendant plus de cinquante ans (1932-1988). L'étude sémiopragmatique menée dans la première partie de cet article n'a conduit ni à une réévaluation de la qualité esthétique des photographies collectées, ni à identifier des aspects particulièrement originaux dans la forme prise par l'interface. Ce constat conduit à considérer que l'intérêt du site Man on Bridge se situe moins au niveau formel, qu'au niveau des fonctionnalités de l'interface et des usages de la part des internautes. Cela mène à adopter six désignations complémentaires (webdocumentaire, documentaire interactif, fonds d'archives en ligne, collection, élément d'un projet transmédia et base de données) de ce site et, ainsi, à trouver un modèle interprétatif adapté à cette forme symptomatique de ce que l'avènement de l'époque du numérique fait à la pratique cinématographique.

\section{AUTHOR}

\section{RÉMY BESSON}

Rémy Besson a soutenu en 2012 un doctorat en histoire à l'EHESS (Paris), portant sur la mise en récit du film Shoah de Claude Lanzmann. Il a été post-doctorant au Centre de Recherches Intermédiales sur les arts, lettres et techniques (CRIalt, Montréal, 2012-2014). Il a été chargé de cours à l'EHESS, à Paris VIII, à l'Université de Montréal et à présent à l'Université de Toulouse. Il est actuellement post-doctorant au LLA-CREATIS (Université de Toulouse II - Jean Jaurès). Il tient sur la plateforme Hypothèses le carnet Cinémadoc. 